\title{
Estruturação da assistência farmacêutica: plano de ação para a seleção de medicamentos essenciais
}

\author{
Structuring of pharmaceutical care: An operational plan for the \\ selection of essential medicines
}

\author{
Rachel Magarinos-Torres ${ }^{1}$, Vera Lucia Edais Pepe², Claudia Garcia Serpa Osorio-de-Castro³
}

\begin{abstract}
Resumo
A adoção da lista de medicamentos essenciais favorece o acesso e contribui com o uso racional de medicamentos à medida em que são incluídos apenas fármacos necessários, com comprovada eficácia, segurança, menor custo tratamento/dia e apresentação mais adequada ao manejo. O texto apresenta um plano de ação para execução da seleção de medicamentos essenciais em esferas governamentais, que pode ser também empregado como instrumento para nortear o processo em instituições de saúde. O desenho metodológico abrigou revisão documental das experiências de seleção de medicamentos da União e dos estados e o exame do caso do estado do Rio de Janeiro. O plano, à luz do referencial teórico da avaliação de serviços, foi organizado em três momentos. A partir do objetivo de cada um, metas e ações necessárias ao seu alcance foram listadas, totalizando 8 metas e 16 ações interligadas e sequenciais. Cada ação é acompanhada pelo detalhamento de sua contribuição para o objetivo, proposta metodológica para realização, recursos necessários, período estimado de execução e indicadores para mensurar se os objetivos específicos foram alcançados. Estima-se que o produto possa subsidiar os processos de seleção em municípios e estados, bem como as revisões periódicas das listas de medicamentos.
\end{abstract}

Palavras-chave: medicamentos essenciais; assistência farmacêutica; gestão em saúde; Brasil.

\begin{abstract}
The adoption of the essential medicines list (EML) enables access to essential medicines and their rational use as only necessary medicines with proven efficacy and safety, favorable cost and dosage forms are included. This work presents an action plan for essential medicines selection in the public health system, which may also be employed as a management tool in health facilities. Methodological design involved a complete review of state experiences in medicines selection, and the analysis of the case in the State of Rio de Janeiro. The plan was developed based on the theoretical framework of health-systems evaluation and organized in a step-wise fashion. For each step, objectives, goals and necessary actions were compiled, totaling three objectives, eight goals and sixteen interlaced actions. Each action, in turn, is accompanied by the detailing of its contribution to the objective, methods, necessary resources, estimated timeframe for completion and indicators for measurement of achievement of objective. We propose that this plan may help subsidize the development of the selection process in states and municipalities, as well as reviews of the EML.
\end{abstract}

Keywords: drugs; essential; pharmaceutical services; health management; Brazil.

Trabalho realizado na Escola Nacional de Saúde Pública Sergio Arouca da Fundação Oswaldo Cruz (FIOCRUZ) - Rio de Janeiro (RJ), Brasil.

'Departamento de Farmácia e Administração Farmacêutica da Faculdade de Farmácia da Universidade Federal Fluminense (UFF) - Niterói (RJ), Brasil.

2Departamento de Administração e Planejamento em Saúde da Escola Nacional de Saúde Pública Sergio Arouca da FIOCRUZ - Rio de Janeiro (RJ), Brasil.

${ }^{3}$ Núcleo de Assistência Farmacêutica da Escola Nacional de Saúde Pública Sergio Arouca da FIOCRUZ - Rio de Janeiro (RJ), Brasil.

Endereço para correspondência: Rachel Magarinos-Torres - Departamento de Farmácia e Administração Farmacêutica - Faculdade de Farmácia -

Universidade Federal Fluminense - Rua Mário Viana, 523 - CEP: 24241-000 - Niterói (RJ), Brasil - E-mail: rmtorres@id.uff.br

Fonte de financiamento: nenhuma.

Conflito de Interesses: nada a declarar. 


\section{INTRODUÇÃO}

A organização da Assistência Farmacêutica (AF), apontada como prioridade para consolidação do sistema de saúde brasileiro, persiste como um desafio para os entes federativos ${ }^{1}$. Segundo a Política Nacional de Medicamentos (PNM), instrumento com que o país conta desde 1998, construído em um longo processo de discussão, a expressão dos compromissos de governo é estabelecida por meio de diretrizes e prioridades, dentre as quais merece destaque a adoção do conceito de medicamentos essenciais ${ }^{2}$.

São também estabelecidas, neste documento, as responsabilidades de cada esfera de governo quanto à organização das atividades relativas aos medicamentos, conferindo as bases para a descentralização da AF. A orientação legal é que o processo de seleção de medicamentos, entendido como base estrutural da $\mathrm{AF}$, aconteça nas diferentes esferas governamentais e níveis de atenção ${ }^{2}$.

A lista de medicamentos essenciais é um instrumento de orientação das condutas clínicas e administrativas para medicamentos em determinado espaço político-social ${ }^{3}$. Sua adoção favorece a ampliação do acesso e contribui com a promoção do uso racional de medicamentos, na medida em que são incluídos apenas fármacos necessários para atender ao perfil epidemiológico da população, e que atendam a requisitos que incluem comprovada eficácia, segurança, menor custo tratamento/dia e apresentação mais adequada ao manejo ${ }^{3,4}$.

A existência da lista nacional de medicamentos essenciais não suprime a necessidade de realização deste processo nos níveis estadual, municipal, em hospitais ou outros serviços públicos ou privados. O que se espera é que haja um fluxo de informação entre esses processos. A lista nacional deve ser entendida como um parâmetro na elaboração das demais listas².

A elaboração das listas estaduais e municipais fortalece o processo de descentralização da gestão e, ao definir necessidades, prioriza e direciona a aplicação de recursos financeiros, humanos e de gestão, das três esferas de governo. Já os movimentos de seleção que acontecem dentro de hospitais e unidades básicas de saúde podem contribuir de forma mais focalizada com informação sobre a demanda e o perfil de utilização, detalhando possibilidades e limites de utilização por profissionais de saúde e pacientes em cenários específicos ${ }^{5}$.

É sabido que a despeito da importância da seleção de medicamentos na proteção da saúde e no acesso aos medicamentos de qualidade, nem sempre a adesão às listas é feita de forma imediata, havendo mesmo dificuldades na compreensão de sua importância. Assim é que, por vezes, ela é tida como instrumento de restrição ao acesso e à decisão dos prescritores e nem sempre ela serve realmente de base para a oferta local de medicamentos ${ }^{4}$. O processo de seleção dos medicamentos pode favorecer, quando bem realizado, uma maior adesão dos profissionais de saúde e mesmo dos gestores à lista, favorecendo o uso racional de medicamentos.

Desde 1999, o Ministério da Saúde tem investido na formação de gestores da AF para o planejamento e organização das atividades administrativas e clínicas. Em 2006, empreendeu um esforço de capacitação que levou aos estados brasileiros 61 seminários de planejamento privilegiando a elaboração de planos de ação para apoiar a implementação das atividades de $\mathrm{AF}^{6}$. No entanto, estima-se que os avanços na estruturação do componente seleção de medicamentos tenham sido tímidos ${ }^{1}$.

Este texto apresenta um plano de ação para apoiar a implementação do processo de seleção de medicamentos essenciais por esferas governamentais com possibilidade de também ser empregado como instrumento norteador deste processo em instituições de saúde.

\section{METODOLOGIA}

$\mathrm{O}$ atendimento ao objetivo de elaboração do plano de ação foi subsidiado e é resultado de dois marcos: o exame por observação direta do caso do estado do Rio de Janeiro e uma revisão documental das experiências publicadas de seleção de medicamentos, da União e dos estados.

O estado do Rio de Janeiro foi examinado como caso explicativo para melhor compreender a realidade e a problemática de seleção de medicamentos em esfera governamental. A escolha foi de conveniência. Em 2009, foi firmado um Projeto de Cooperação entre a Secretaria de Estado de Saúde e Defesa Civil do Estado do Rio de Janeiro (SESDEC) e a Escola Nacional de Saúde Pública Sérgio Arouca (ENSP-FIOCRUZ) com uma linha coordenada pelo Núcleo de Assistência Farmacêutica (NAF). O delineamento desta parte do projeto, denominado Apoio à Organização da Assistência Farmacêutica no estado do Rio de Janeiro, foi realizado em conjunto com a SESDEC. Foram definidos cinco eixos estratégicos para o projeto, sendo o primeiro a Seleção de Medicamentos no estado do Rio de Janeiro - Lista Estadual de Medicamentos Essenciais (LEME-RJ). A parceria propiciou aproximação com o caso e facilitou o acesso aos dados necessários à realização da pesquisa.

A coleta de dados desta etapa foi realizada por observação participante. Entre agosto e dezembro de 2009 foram realizadas três reuniões presenciais com a equipe da Superintendência de Assistência Farmacêutica e Insumos Estratégicos (SAFIE/SESDEC). Duas outras reuniões, da Comissão Estadual de Farmácia e Terapêutica do Estado do Rio de Janeiro (CFT-RJ), em trabalho de seleção, foram alvo 
de observação. Além disto, foram recuperadas e analisadas sete de nove atas de reuniões da CFT-RJ que aconteceram entre março e agosto de 2009. Os dados coletados foram empregados na descrição do cenário que levou à elaboração do plano de ação para a implementação do processo de seleção de medicamentos.

As experiências de seleção de medicamentos da União e dos estados foram identificadas a partir da localização e análise dos documentos que apresentam as listas de medicamentos essenciais publicadas. Como premissa considerou-se que a divulgação de lista de medicamentos essenciais é parte do processo de seleção. É esperado que, caso haja uma lista de medicamentos essenciais, esta seja amplamente divulgada. A lista é também um indicador sentinela dos processos realizados.

A internet foi o veículo de comunicação eleito para determinação da publicação da lista. Foram consultados os sites do Ministério da Saúde, do Distrito Federal e das Secretarias Estaduais de Saúde dos 26 estados brasileiros. Considerando o papel da lista de medicamentos essenciais na organização da AF, ela deve estar disponível no site das Secretarias de Saúde muito possivelmente ligada ao grupo coordenador das ações de AF daquele âmbito. Nos casos não localizados por esta estratégia, foi realizada busca direta com ferramenta localizadora de internet, usando os termos "Lista Estadual de Medicamentos" e "Relação Estadual de Medicamentos" acrescido do nome de cada um dos estados. A busca foi realizada em novembro de 2009.

A análise dos documentos buscou identificar os aspectos metodológicos adotados no processo de seleção e o modo de estruturação/apresentação, publicação e divulgação da lista. As estratégias utilizadas para convidar e estimular os envolvidos na preparação do processo, a busca e a análise das evidências no momento de escolha dos itens compõem os aspectos metodológicos, focalizando os critérios adotados e a manutenção do processo de trabalho.

Foi definida a estrutura do plano de ação. O processo de seleção, à luz do referencial teórico da avaliação de serviços, foi caracterizado em três momentos ${ }^{7}$. O primeiro, referente à estrutura, onde são executadas ações relacionadas ao planejamento e preparação do processo; o segundo, de análise propriamente dita dos itens, entendida como o cerne do processo de seleção e o terceiro, o resultado, onde são alocadas as ações de implementação da lista e de monitoramento visando adesão à relação de medicamentos construída. A cada dimensão foram relacionados objetivos específicos e, a partir desses, metas e ações correspondentes, com base em referências de planejamento da AF. Cada ação é acompanhada pelo detalhamento de sua contribuição para o objetivo, uma proposta operacional incluindo os recursos necessários, o período estimado de execução e indicadores para mensurar se os objetivos específicos foram alcançados.

\section{RESULTADOS E DISCUSSÃO}

Integram o plano de ação 8 metas e 16 ações interligadas e sequenciais construídas a partir dos 3 momentos definidos a priori (Quadro 1).

O cenário observado no estado do Rio de Janeiro apontou as ações necessárias ao momento de planejamento e preparação do plano de ação. A observação trouxe a necessidade de definir metas relacionadas ao estabelecimento do grupo de trabalho, do fluxo de trabalho e elaboração da lista preliminar ${ }^{8}$.

No momento investigado, o estado do Rio de Janeiro trabalhava a seleção de medicamentos de modo fragmentado. Coexistiam duas comissões de farmácia e terapêutica, ambas designadas por portaria estadual, uma com o propósito de construir a lista de medicamentos da Atenção Básica e a outra voltada para a revisão da relação de medicamentos necessários às unidades de gestão direta da SESDEC. Esta opção metodológica não abrigava a maior parte das necessidades dos pacientes atendidos na rede de saúde do estado e pouco colaborava na identificação das prioridades ou no direcionamento de recursos. Na seleção, enquanto análise comparativa dos medicamentos, é importante observar todos os itens para uma mesma indicação confrontando os dados de eficácia, segurança e os recursos para sua utilização ${ }^{8-10}$.

Sobre as duas comissões de farmácia e terapêutica identificadas no estado do Rio Janeiro cabe colocar que a coordenação de ambas era da SAFIE/SESDEC. A composição daquela destinada ao elenco da Atenção Básica privilegiava a participação das Macrorregiões de Saúde, na figura dos gestores municipais de AF, enquanto a outra, voltada ao elenco das unidades de gestão direta da SESDEC, era composta por farmacêuticos dos hospitais estaduais ${ }^{8-10}$.

É interessante que a composição do grupo de trabalho seja feita de forma a contemplar as áreas de conhecimento imprescindíveis na elaboração da lista. A qualidade das etapas seguintes como a análise propriamente dita dos itens depende de conhecimentos das áreas de Farmacologia Clínica; Saúde Pública com ênfase em Políticas Farmacêuticas; Farmacoepidemiologia envolvendo a utilização, a gestão de riscos e custos; Medicina Clínica, Pesquisa Clínica, Tecnologia Farmacêutica, Avaliação em Saúde com ênfase em Avaliação Tecnológica e Econômica ${ }^{11}$.

Para tanto, devem ser identificados potenciais membros com liderança e capacidade técnica para, em seguida, convidar e oficializar o grupo, por meio de ato legislativo. 
Quadro 1. Plano de ação a seleção de medicamentos essenciais

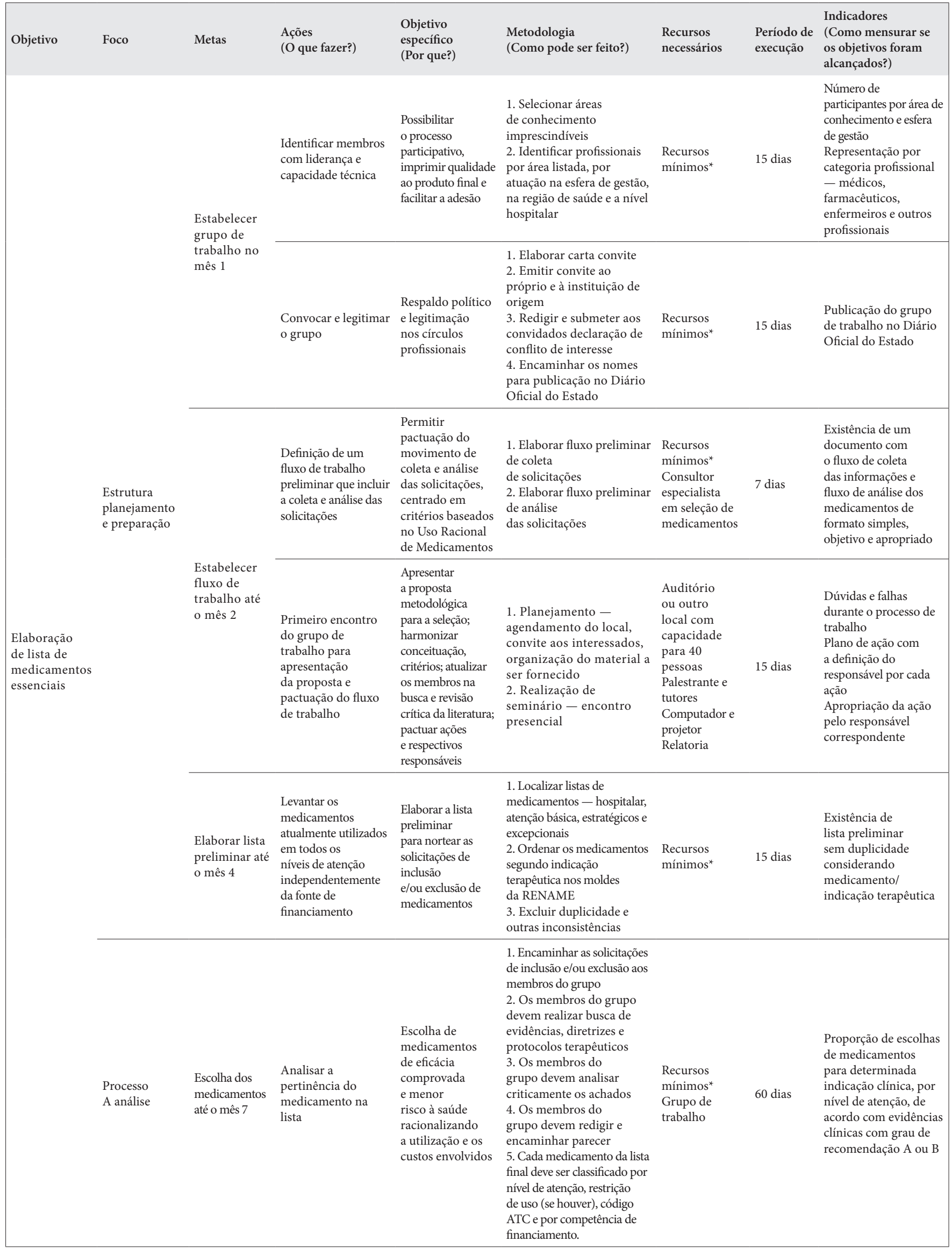


Quadro 1. Continuação

\begin{tabular}{|c|c|c|c|c|c|c|c|c|}
\hline Objetivo & Foco & Metas & $\begin{array}{l}\text { Ações } \\
\text { (O que fazer?) }\end{array}$ & $\begin{array}{l}\text { Objetivo } \\
\text { específico } \\
\text { (Por que?) }\end{array}$ & Metodologia (Como pode ser feito?) & $\begin{array}{l}\text { Recursos } \\
\text { necessários }\end{array}$ & $\begin{array}{l}\text { Período } \\
\text { de } \\
\text { execução }\end{array}$ & $\begin{array}{l}\text { Indicadores } \\
\text { (Como mensurar se } \\
\text { os objetivos foram } \\
\text { alcançados?) }\end{array}$ \\
\hline \multirow{9}{*}{$\begin{array}{l}\text { Elaboração } \\
\text { de lista de } \\
\text { medicamentos } \\
\text { essenciais }\end{array}$} & \multirow{9}{*}{$\begin{array}{l}\text { Resultado } \\
\text { Implementação } \\
\text { e } \\
\text { monitoramento } \\
\text { Promoção da } \\
\text { adesão }\end{array}$} & \multirow{4}{*}{$\begin{array}{l}\text { Divulgação } \\
\text { até } \\
\text { o mês } 9\end{array}$} & $\begin{array}{l}\text { Organização } \\
\text { do documento } \\
\text { final }\end{array}$ & $\begin{array}{l}\text { Facilitar consulta } \\
\text { e identificação } \\
\text { do medicamento, } \\
\text { respectiva forma } \\
\text { farmacêutica, } \\
\text { restrição de uso } \\
\text { e competência } \\
\text { na aquisição e } \\
\text { distribuição }\end{array}$ & $\begin{array}{l}\text { 1. Reunir documentos utilizados - } \\
\text { formulários, normas e pareceres do grupo } \\
\text { de trabalho } \\
\text { 2. Reunir os medicamentos eleitos } \\
\text { 3. Elaborar o documento final - } \\
\text { informações preliminares, medicamentos } \\
\text { organizados de acordo com a RENAME } \\
\text { e apêndices } \\
\text { 4. Encaminhar ao revisor }\end{array}$ & $\begin{array}{l}\text { Recursos } \\
\text { mínimos* } \\
\text { Editor } \\
\text { Revisor }\end{array}$ & 15 dias & $\begin{array}{l}\text { Percepção de gestores e } \\
\text { prescritores a respeito do } \\
\text { formato do documento } \\
\text { Dificuldades de } \\
\text { utilização apresentadas } \\
\text { por gestores e } \\
\text { prescritores }\end{array}$ \\
\hline & & & $\begin{array}{l}\text { Homologação } \\
\text { da lista }\end{array}$ & $\begin{array}{l}\text { Respaldo político } \\
\text { e legitimação }\end{array}$ & $\begin{array}{l}\text { 1. Encaminhar a lista para publicação em } \\
\text { Diário Oficial }\end{array}$ & $\begin{array}{l}\text { Recursos } \\
\text { mínimos }\end{array}$ & 7 dias & $\begin{array}{l}\text { Publicação da lista no } \\
\text { Diário Oficial }\end{array}$ \\
\hline & & & $\begin{array}{l}\text { Publicação e } \\
\text { distribuição }\end{array}$ & $\begin{array}{l}\text { Possibilitar } \\
\text { acesso ao } \\
\text { documento }\end{array}$ & $\begin{array}{l}\text { 1. Publicação em formato eletrônico e } \\
\text { em papel } \\
\text { Distribuição gratuita em papel e em mídia } \\
\text { eletrônica }(C D)\end{array}$ & $\begin{array}{l}\text { Recursos } \\
\text { mínimos* } \\
\text { Web designer } \\
\text { Gráfica }\end{array}$ & 15 dias & $\begin{array}{l}\text { Porcentagem de } \\
\text { instituições de saúde que } \\
\text { receberam o documento } \\
\text { Prescritores e } \\
\text { dispensadores que } \\
\text { receberam o documento }\end{array}$ \\
\hline & & & Divulgação & $\begin{array}{l}\text { Disseminação } \\
\text { da finalidade e } \\
\text { utilização }\end{array}$ & $\begin{array}{l}\text { 1. Lançamento oficial em evento com } \\
\text { cobertura por meios de comunicação } \\
\text { 2. Divulgação na internet e em outros } \\
\text { meios de comunicação } \\
\text { 3. Carta e cartazes a instituições de saúde } \\
\text { e organizações profissionais } \\
\text { 4. Encontros educacionais interativos } \\
\text { 5. Proposição de sistemas } \\
\text { computadorizados para nortear a } \\
\text { prescrição, a gestão de estoques, o } \\
\text { fornecimento e a administração }\end{array}$ & $\begin{array}{l}\text { Recursos } \\
\text { mínimos* } \\
\text { Local para } \\
\text { divulgação e } \\
\text { encontros } \\
\text { Especialistas } \\
\text { em computação } \\
\text { para elaboração } \\
\text { dos sistemas } \\
\text { computadorizados }\end{array}$ & 30 dias & $\begin{array}{l}\text { Número de ações de } \\
\text { disseminação ativa } \\
\text { Conhecimento de } \\
\text { gestores e prescritores } \\
\text { estaduais, sobre a lista, } \\
\text { em periodicidade regular }\end{array}$ \\
\hline & & $\begin{array}{l}\text { Elaboração } \\
\text { do } \\
\text { formulário } \\
\text { terapêutico } \\
\text { até } \\
\text { o mês } 10\end{array}$ & $\begin{array}{l}\text { Elaborar o } \\
\text { Formulário } \\
\text { Terapêutico }\end{array}$ & $\begin{array}{l}\text { Fornecer } \\
\text { informação } \\
\text { sobre os } \\
\text { medicamentos, } \\
\text { o uso destes em } \\
\text { condições de } \\
\text { saúde específicas, } \\
\text { sobre políticas e } \\
\text { procedimentos } \\
\text { que norteiam } \\
\text { a prescrição, o } \\
\text { armazenamento, } \\
\text { o fornecimento e } \\
\text { a administração. }\end{array}$ & $\begin{array}{l}\text { 1. Construir as monografias - } \\
\text { informação objetiva, apropriada e } \\
\text { independente - pode-se apoiar no } \\
\text { Formulário Terapêutico Nacional (FTN) } \\
\text { - a construção das monografias pode ser } \\
\text { distribuída pelo grupo de trabalho } \\
\text { 2. Redigir informações gerais para } \\
\text { determinada condição de saúde e/ou } \\
\text { grupo de medicamentos }\end{array}$ & $\begin{array}{l}\text { Recursos } \\
\text { mínimos } \\
\text { Grupo de trabalho } \\
\text { Colaboradores }\end{array}$ & 30 dias & $\begin{array}{l}\text { Formulário terapêutico } \\
\text { com informações sobre os } \\
\text { medicamentos da lista, as } \\
\text { políticas e procedimentos } \\
\text { para a prescrição, } \\
\text { o armazenamento, } \\
\text { o fornecimento e a } \\
\text { administração na esfera } \\
\text { administrativa }\end{array}$ \\
\hline & & \multirow{3}{*}{$\begin{array}{l}\text { Divulgação } \\
\text { do } \\
\text { Formulário } \\
\text { Terapêutico } \\
\text { até o mês } \\
12\end{array}$} & $\begin{array}{l}\text { Organização } \\
\text { do documento } \\
\text { final }\end{array}$ & & $\begin{array}{l}\text { 1. Reunir as monografias elaboradas } \\
\text { 2. Elaborar o documento final - } \\
\text { informações preliminares, seções } \\
\text { específicas com informações para } \\
\text { determinadas condições de saúde e/ou } \\
\text { grupo de medicamentos, monografias dos } \\
\text { medicamentos e apêndices } \\
\text { 3. Encaminhar ao revisor }\end{array}$ & $\begin{array}{l}\text { Recursos } \\
\text { mínimos* } \\
\text { Editor } \\
\text { Revisor }\end{array}$ & 21 dias & $\begin{array}{l}\text { Percepção dos profissionais } \\
\text { de saúde a respeito do } \\
\text { formato do documento } \\
\text { Dificuldades de utilização } \\
\text { apresentadas por } \\
\text { profissionais de saúde }\end{array}$ \\
\hline & & & $\begin{array}{l}\text { Publicação e } \\
\text { distribuição }\end{array}$ & & $\begin{array}{l}\text { 1. Publicação em formato eletrônico e } \\
\text { em papel } \\
\text { Distribuição gratuita em papel e em mídia } \\
\text { eletrônica }(C D)\end{array}$ & $\begin{array}{l}\text { Recursos } \\
\text { mínimos* } \\
\text { Web designer } \\
\text { Gráfica }\end{array}$ & 15 dias & $\begin{array}{l}\text { Porcentagem de } \\
\text { instituições de saúde que } \\
\text { receberam o formulário } \\
\text { terapêutico } \\
\text { Prescritores e } \\
\text { dispensadores que } \\
\text { receberam o formulário } \\
\text { terapêutico }\end{array}$ \\
\hline & & & Divulgação & & $\begin{array}{l}\text { 1. Lançamento oficial em evento com } \\
\text { cobertura por meios de comunicação } \\
\text { 2. Divulgação na internet e em outros } \\
\text { meios de comunicação } \\
\text { 3. Carta e cartazes à instituições de saúde } \\
\text { e organizações profissionais } \\
\text { 4. Encontros educacionais interativos }\end{array}$ & $\begin{array}{l}\text { Recursos } \\
\text { mínimos* } \\
\text { Local para } \\
\text { divulgação e } \\
\text { encontros }\end{array}$ & 30 dias & $\begin{array}{l}\text { Número de ações de } \\
\text { disseminação ativa }\end{array}$ \\
\hline & & $\begin{array}{l}\text { Avaliação } \\
\text { dos } \\
\text { resultados } \\
\text { entre os } \\
\text { meses } 18 \\
\text { e } 24\end{array}$ & $\begin{array}{l}\text { Investigar as } \\
\text { consequências } \\
\text { para os } \\
\text { pacientes, } \\
\text { profissionais } \\
\text { e sistema de } \\
\text { saúde }\end{array}$ & $\begin{array}{l}\text { Fornecer dados } \\
\text { que permitam a } \\
\text { reorientação das } \\
\text { procedimentos } \\
\text { adotados para } \\
\text { revisão da lista }\end{array}$ & $\begin{array}{l}\text { 1. Investigar se houve aumento de } \\
\text { cobertura, redução de eventos adversos } \\
\text { relacionados a medicamentos, redução } \\
\text { de compras de urgência, redução de } \\
\text { processos judiciais relacionados a } \\
\text { medicamentos, melhora no processo de } \\
\text { trabalho nas unidades de saúde, redução } \\
\text { de despesas }\end{array}$ & $\begin{array}{l}\text { Pesquisador } \\
\text { com experiência } \\
\text { em Assistência } \\
\text { Farmacêutica }\end{array}$ & 180 dias & $\begin{array}{l}\text { Relatório capaz de } \\
\text { subsidiar a revisão da lista }\end{array}$ \\
\hline
\end{tabular}


Estas ações fortalecem o caráter participativo do processo, conferem respaldo político e legitimação nos círculos profissionais, imprimem qualidade ao produto final, e facilitam a adesão à lista quando publicada ${ }^{12}$.

O próximo passo apontado no plano de ação é identificar dois profissionais por área de conhecimento. $\mathrm{O}$ esperado é que a identificação por área consiga compor um grupo multidisciplinar com a presença de pelo menos um médico e um farmacêutico com vivência em prescrição e fornecimento de medicamentos em espaços de saúde ${ }^{5}$. Caso isto não ocorra, é interessante convidar estes atores, dada a importância de selecionar considerando a aplicabilidade das decisões no cotidiano profissional. Por motivo semelhante, é desejável a presença de representantes das esferas de gestão estadual e municipal.

Devem também ser convidados dois representantes da esfera hospitalar, dada à complexidade dos problemas de saúde enfrentados nesse ambiente, bem como, a necessidade de utilização de medicamentos distintos daqueles incluídos nos componentes de financiamento e em listas pré-pactuada com as esferas municipal ou estadual.

Uma vez identificados, antes da publicação no Diário Oficial, o grupo deve ser convocado e os possíveis membros devem declarar seus interesses de forma a se identificar e classificar possíveis conflitos. Os medicamentos têm importante valor comercial. O contato propagandístico da indústria farmacêutica com profissionais de saúde tem início quando estes ainda estão na graduação, afetando o seu comportamento frente a novas tecnologias ${ }^{13}$. A existência de vínculo com os setores comerciais é perigosa para a seleção de medicamentos essenciais e compromete a independência e imparcialidade indispensáveis às ações de uma Comissão de Farmácia e Terapêutica 5 .

O momento de planejamento e preparação abriga, ainda, a elaboração da lista preliminar e o estabelecimento do fluxo de trabalho ${ }^{5}$. A construção da lista preliminar tem o propósito de nortear a análise que dá origem às solicitações de inclusão ou exclusão de um determinado item. Devem ser identificados os medicamentos utilizados nos hospitais e institutos de gestão direta, na atenção básica, e os presentes na lista de estratégicos e excepcionais. Este conjunto deve ser ordenado segundo indicação terapêutica. Em seguida, devem ser excluídas as duplicidades e outras inconsistências identificadas. Esta ação é desnecessária quando se trata da revisão da lista de medicamentos. Outra possibilidade, quando da elaboração da primeira versão, é adotar a relação nacional e a partir dela identificar necessidades do estado.

A inclusão desta etapa no plano de ação encontra sentido nos achados da revisão bibliográfica e no caso do Rio de Janeiro. Foram localizadas as seguintes listas estaduais de medicamentos essenciais: Amazonas ${ }^{17}$, Bahia ${ }^{15}$, Espírito Santo ${ }^{14}$ e Goiás ${ }^{16}$.
Todas na primeira edição, ou seja, poucos são os estados brasileiros que possuem relação de medicamentos essenciais. O estado do Rio de Janeiro, por sua vez, nunca teve uma lista única de medicamentos essenciais capaz de apoiar uma revisão. A comissão que trabalhava a construção do elenco para as unidades de gestão direta da SESDEC adotava como 'lista base' todos os itens distribuídos na rede ${ }^{14-17}$.

Quanto ao processo de trabalho, o plano traz ações destinadas a estruturar o processo de modo a concluir com qualidade, em um prazo de 90 dias, a análise da pertinência dos medicamentos na lista. A Comissão Estadual de Farmácia e Terapêutica do Estado do Rio de Janeiro utilizou 6 meses para conclusão de 2 dos 19 grupos inicialmente listados: anestésicos e antimicrobianos. A maior parte do tempo das reuniões presenciais foi utilizada para discutir o fluxo de abastecimento e problemas de desabastecimento dessas unidades, a qualidade dos produtos adquiridos e armazenados e a gestão e capacitação de recursos humanos na farmácia hospitalar. Embora seja perceptível a preocupação com a qualidade do processo de seleção, as atas de reunião não traziam o fluxo de trabalho ou o calendário das atividades; tampouco exposição dos critérios utilizados na escolha dos medicamentos. Aparentemente, o processo utilizou poucos recursos de busca de evidência ${ }^{8}$.

Na seleção, o fluxo de trabalho diz respeito à coleta e análise das solicitações de inclusão ou exclusão de medicamentos na lista ${ }^{5}$. O termo "fluxo de trabalho" será aqui utilizado para designar uma sequência de passos envolvendo as atividades e as regras de procedimento. Foram listadas como ações necessárias à elaboração de um fluxo preliminar e um momento presencial para discutir a proposta, harmonizar conceituação e critérios, pactuar ações e respectivos responsáveis. O encontro presencial pode ser utilizado, também, para atualizar os membros na busca e revisão crítica da literatura científica relacionada a medicamentos. A qualidade do processo de seleção depende, em muito, da postura crítica dos membros frente à literatura disponível.

A definição do elenco de medicamentos deve ser balizada por critérios que permitam escolher de forma racional considerando os benefícios frente aos riscos e custos envolvidos ${ }^{3}$. O documento final deve expor as atividades, os critérios que serão utilizados na escolha dos medicamentos e a competência dos membros ${ }^{11}$.

A proposta para o momento presencial sugerido é de um seminário, com exposições seguidas de debates, conduzidos por um ou mais especialistas no assunto, com duração total de oito horas, sendo parte dedicadas à apresentação oral da base teórica e parte destinadas à pactuação do processo de trabalho. As seguintes temáticas merecem ser abordadas: o papel da seleção de medicamentos no cuidado individual e no sistema de saúde, conceitos de eficácia e segurança de medicamentos, a epidemiologia no julgamento das publicações e na coleta de 
evidências de segurança pós-comercialização e a construção e utilização de instrumentos de normalização, como as listas de medicamentos, protocolos e diretrizes terapêuticas.

Espera-se que o encontro presencial seja capaz de estimular a participação dos membros nas diversas fases do processo de seleção; é importante sensibilizar e mobilizar a instituição que representam para o envio de solicitações de inclusão e exclusão de medicamentos. Também é desejável que o próprio membro encaminhe suas sugestões quanto à composição da lista ${ }^{11}$. O comprometimento dos membros e da instituição tem papel fundamental na implementação e monitoração dos resultados, onde eles serão atores importantes na elaboração e divulgação do formulário terapêutico ${ }^{18}$.

O formulário de solicitação de inclusão ou exclusão e a lista preliminar devem ser publicados na forma de consulta pública. Este documento legal deve expor o prazo máximo para envio das solicitações. São consideradas como solicitação colocações relacionadas com a nomenclatura (Denominação Comum Brasileira - DCB), apresentação, concentração, dose e/ou usos terapêuticos.

A estruturação do processo de trabalho discutida acima tem origem na revisão bibliográfica dos processos de seleção conduzidos pela esfera federal. Depois da publicação da Política Nacional de Medicamentos em 1998, a Relação Nacional de Medicamentos Essenciais (RENAME) foi revista em cinco momentos: 2000, 2002, 2006, 2008 e 2010. Em todos, o processo foi conduzido por uma comissão multidisciplinar, sendo os dois últimos por um grupo de trabalho institucionalizado com regimento e composição pré-estruturada denominado de Comissão Técnica e Multidisciplinar de Atualização da RENAME (COMARE).

A partir de 2006, o processo de revisão da RENAME passa por uma qualificação cada vez maior, o que pode ser observado pela institucionalização da revisão, pela organização do processo em si (regimento, composição, cronograma de reuniões, fluxos de trabalho, exame de questões éticas), pelo rigor metodológico presente na revisão (treinamentos para busca de evidências, padronização de pareceres) e pelo amadurecimento do processo de revisão, o que ocasionou o surgimento de outro produto, o Formulário Terapêutico Nacional ${ }^{19-24}$.

Para a análise quanto à pertinência dos itens na lista de medicamentos essenciais, as solicitações de inclusão ou exclusão encaminhadas devem ser observadas comparativamente por indicação clínica. A definição de condutas racionais passa pela comparação entre os benefícios, os riscos e os gastos na adoção de diferentes estratégias. Estes critérios podem estar associados às características intrínsecas dos medicamentos ou aos procedimentos que eles demandariam ao serviço. Por exemplo, a inclusão de um medicamento que precise de um espaço diferenciado para armazenamento ou que apresente dissolução não usual - inclusões que ensejam a possível introdução de novos procedimentos ou que favoreçam a ocorrência de erros de medicação relacionados à administração.

São critérios principais a serem considerados, a eficácia e a segurança. Somente depois de analisadas as evidências e, tendo estas respondido à pergunta clínica, é que as implicações práticas da inserção ou exclusão de um item na lista serão consideradas. Atendidos os critérios acima, outros tais como disponibilidade, facilidade de adesão e sustentabilidade econômica merecem ser considerados ${ }^{25}$.

Como evidências, valorizam-se os estudos científicos bem conduzidos que não sejam permeados por interesses comerciais no produto. $\mathrm{O}$ formulário de análise deve apontar para uso de revisões sistemáticas, e para a qualidade e força da evidência resgatada. Segundo Wannmacher ${ }^{23}$,

A qualidade da evidência indica a extensão de sua confiabilidade e se a estimativa de efeito está correta. A força da evidência indica sua relevância clínica e aplicabilidade, ou seja, a capacidade de se ajustar à prática clínica e a estimativa de que a recomendação por ela gerada tenha mais benefício do que risco.

Em seguida, deve ser elaborado e encaminhado à coordenação do processo um parecer técnico expondo as razões da exclusão, inclusão ou alteração de apresentação, concentração, dose e/ou usos terapêuticos. O período de execução estipulado no plano de ação para as análises foi de 60 dias, calculado na perspectiva de que cada membro tenha o prazo máximo de uma semana por análise.

A última ação inserida nesta etapa é a classificação dos medicamentos por nível de atenção, por restrição de uso (se houver), pela classificação "Código Anatômico, Terapêutico e Químico" (código ATC) ${ }^{26}$ e por competência de financiamento na aquisição e na distribuição ${ }^{27-29}$. O nível de atenção faz referência à atenção primária, à média e à alta complexidade. Cabe restrição de uso em casos com potencial grave de risco para o paciente ou para o coletivo, medicamentos com indicação para circunstâncias bem específicas ou, que, em razão de seu elevado custo, devem ser reservados para as indicações em que se constituem tratamento de primeira escolha. A competência diz respeito à responsabilidade pela aquisição e pelo fornecimento, podendo ser, por exemplo, medicamentos com aquisição pelos municípios e dispensação nas Unidades Básicas de Saúde, ou ainda, medicamentos para pacientes internados nos hospitais da rede estadual, cuja aquisição e distribuição são de responsabilidade de cada Unidade. A proposta, enunciada com base na observação das listas do Espírito Santo e da Bahia, é que a lista forneça estas informações por medicamento ${ }^{14,15}$. 
A próxima etapa, de implementação e monitoramento, aparece no plano de ação subdividido em 4 metas, a saber: divulgação até o mês 9 , elaboração do formulário terapêutico até o mês 10 , divulgação do formulário até o mês 12, e avaliação da implementação entre os meses 18 e 24.

O propósito da divulgação é promover a adesão, ou seja, a utilização dos medicamentos selecionados respeitando as respectivas e citadas indicações e esquemas de administração, com atenção às contraindicações, às precauções, aos aspectos farmacocinéticos e farmacêuticos e aos possíveis efeitos adversos e interações medicamentosas ${ }^{5}$.

A divulgação com vistas à disseminação da finalidade e da utilização pode envolver evento oficial de lançamento dos documentos, inserção destes em sites qualificados da internet, distribuição de carta e cartazes a instituições de saúde e organizações profissionais e em encontros educacionais e gerenciais. A literatura recente tem destacado a imensa contribuição dos sistemas computadorizados para nortear a prescrição, a gestão de estoques, o fornecimento e a administração ${ }^{30}$. A construção de um sistema computadorizado a partir da lista pode auxiliar muito na adesão à mesma. Cabe levantar a capacidade das unidades no tocante aos recursos estruturais para receber esta tecnologia.

O formulário terapêutico foi entendido como um componente inseparável do processo de seleção e facilitador da adesão à lista. Tanto a lista como o formulário terapêutico merecem ser publicados em papel e em formato eletrônico (CD) e, distribuídos gratuitamente às unidades e profissionais de saúde, especialmente para prescritores, enfermeiros e farmacêuticos.

A elaboração do formulário terapêutico pode se apoiar no formulário terapêutico nacional ${ }^{23}$; com os devidos ajustes quanto a demandas específicas, às políticas e procedimentos que norteiam a prescrição, o armazenamento, o fornecimento e a administração em determinada esfera. A tarefa de revisão e elaboração das monografias pode ser atribuída aos integrantes da comissão. O plano de ação prevê, nesta etapa, a inserção de colaboradores para que o prazo estipulado de 30 dias seja cumprido.

A última meta desta etapa trata do monitoramento. Considera-se vital levantar dados que permitam a reorientação dos procedimentos adotados para balizar a revisão da lista. O processo de seleção é dinâmico e tanto a lista quanto o formulário merecem ser revistos com periodicidade ${ }^{3}$.

A avaliação considera se os objetivos específicos de cada ação listada no plano foram alcançados e como isto se deu, bem como os resultados para os pacientes, profissionais e sistema de saúde. Neste contexto, podem ser investigados, por exemplo, aumento de cobertura, redução de eventos adversos relacionados a medicamentos, redução de compras de urgência, redução de processos judiciais relacionados a medicamentos, melhora no processo de trabalho nas unidades de saúde e redução de despesas.

A realização das atividades necessárias para atingir as metas acima listadas depende da existência durante toda a execução do plano de ação de uma coordenação contendo pelo menos um profissional com disponibilidade de 40 horas semanais e competência técnica para secretariar, espaço físico adequado para este profissional, linha telefônica exclusiva, computador com acesso à internet, impressora, material de escritório e despesas de correio. Este conjunto estrutural foi designado no plano de ação de recursos mínimos.

\section{CONSIDERAÇÕES FINAIS}

O plano de ação proposto foi construído de sorte a dar resposta à necessidade de organizar a $\mathrm{AF}$ a partir da seleção de medicamentos. Esta etapa da AF é muito importante para as unidades e sistemas de saúde, do momento que esses insumos quando mal utilizados, são responsáveis por riscos e danos aos usuários. Administrativamente, representam uma despesa direta considerável e, no caso de danos possíveis, trarão despesas de ordem indireta, representadas por custos de internação e de tratamentos das sequelas do uso.

O Brasil tem avançado com as revisões da lista nacional de medicamentos essenciais, mostrando acúmulo de experiência e amadurecimento do processo de seleção em nível federal. No entanto, as evidências colhidas dos processos e produtos da seleção em diversos estados da União, entre os quais o Rio de Janeiro, mostram que existem lacunas importantes nas atividades e desafios a superar. Fica evidente que a experiência nacional não tem subsidiado suficientemente os estados; por outro lado, é possível que esses entes também não tenham se mobilizado para atender às demandas necessárias a um completo e adequado processo de seleção. É admissível supor que a informação do processo executado com sucesso no nível federal e em algumas instâncias estaduais não esteja atingindo os demais níveis organizacionais da AF.

Neste sentido, a conformação de um plano de ação estruturado, baseado em evidências empíricas e focado na seleção de medicamentos, parece ser peça chave para apoiar a organização de serviços farmacêuticos no âmbito do Sistema Único de Saúde.

\section{REFERÊNCIAS}

1. Vieira FS. Qualificação dos serviços farmacêuticos no Brasil: aspectos inconclusos da agenda do Sistema Único de Saúde. Rev Panam Salud Publica. 2008;24(2):91-100.
2. Brasil. Ministério da Saúde. Secretaria de Políticas de Saúde. Departamento de Atenção Básica. Política Nacional de Medicamentos. Ministério da Saúde (Série C. Projetos, Programas e Relatórios, n. 25), 2001. 40 p. 
3. World Health Organization (WHO). The selection of essential medicines. Geneva: WHO, 2002. p. 1-6.

4. Pepe VLE, Osorio-de-Castro CGS, Luiza VL. A Relação Nacional de Medicamentos Essenciais: um instrumento da Política Nacional de Medicamentos na garantia do acesso. In: Projeto Inovação em Saúde. Presidência FIOCRUZ (Org.). Inovação, pesquisa e acesso a medicamentos no Brasil. Rio de Janeiro: FIOCRUZ, 2008. p. 319-34.

5. Laing R, Tisocki K. How to develop a national formulary based on the WHO model formulary: a practical guide. Geneva: World Health Organization, 2004. 45 p.

6. Brasil. Ministério da Saúde. Secretaria de Ciência, Tecnologia e Insumos Estratégicos. Departamento de Assistência Farmacêutica e Insumos Estratégicos. Planejar é preciso: uma proposta de método para aplicação à assistência farmacêutica (Série B. Textos Básicos de Saúde). Brasília: Editora do Ministério da Saúde, 2006. 74 p.

7. Donabedian A. The role of outcomes in quality assessment and assurance. QRB Qual Rev Bull. 1992;18(11):356-60.

8. Magarinos-Torres R. Eixo 01 - Seleção de medicamentos no Estado do Rio de Janeiro - Lista Estadual de Medicamentos Essenciais (LEME - RJ). In: Osorio-de-Castro CGS, Luiza VL (orgs). Relatório Técnico do Projeto de Cooperação - Apoio à organização da Assistência Farmacêutica no Estado do Rio de Janeiro. ENSP - SESDEC, 2008, p. 226.

9. Rio de Janeiro (estado). Resolução SESDEC n 286 de 09 de maio de 2008. Cria a Comissão Estadual de Farmácia e Terapêutica e dá outras providências. Diário Oficial do Estado de 17 de maio de 2008.

10. Rio de Janeiro (estado). Resolução SESDEC no 329 de 25 de junho de 2008. Renomeia a Comissão Estadual de Assistência Farmacêutica. Diário Oficial do Estado no 117 de 30 de junho de 2008.

11. Brasil. Ministério da Saúde. Departamento de Assistência Farmacêuticas e Insumos Estratégicos. Relação Nacional de Medicamentos Essenciais RENAME 2010. (Série B. Textos Básicos de Saúde). Brasília: Ministério da Saúde, 2010.

12. Marques DC, Zucchi P. Comissões farmacoterapêuticas no Brasil: aquém das diretrizes internacionais. Rev Panam Salud Publica. 2006;19(1):58-63.

13. Palácios M, Rego S, Lino MH. Promoção e propaganda de medicamentos em ambientes de ensino: elementos para o debate. Interface (Botucatu). 2008;12(27):893-905.

14. Espírito Santo (estado). Secretaria de Estado da Saúde. Gerência de Assistência Farmacêutica. Relação Estadual de Medicamentos Essenciais e Medicamentos Excepcionais - REMEME. Vitória, 2007. 342 p.

15. Bahia (estado). Secretária da Saúde do Estado da Bahia. Relação Estadual de Medicamentos Essenciais. Versão para consulta pública. Disponível em: http://www2.saude.ba.gov.br/cib/arquivos/ Apresenta\%C3\%A7\%C3\%B5es\%20das\%20Reuni\%C3\%B5es\%20da\%20 CIB/2008/F.\%20Junho/Apresenta\%C3\%A7\%C3\%A3o\%20DASF/ RESME.pdf. Acesso em out. 2009. 21 p.

16. Goiás (estado). Secretaria de Estado da Saúde. Superintendência de Políticas e Atenção Integral a Saúde. Sub gerência de Assistência Farmacêutica. Relação Estadual de Medicamentos Essenciais - RESME-GO. Organização Pan-Americana de Saúde. Goiânia - GO. 2a ed., 2006. 133 p.
17. Amazonas (estado). Secretaria de Estado da Saúde. Gerência de Assistência Farmacêutica. Manaus, dez. 2003. 41 p.

18. Cabana MD, Rand CS, Powe NR, Wu AW, Wilson MH, Abboud P, et al. Why don't physicians follow clinical practice guidelines? A framework for improvement. JAMA. 1999;282(15):1458-65.

19. Brasil. Ministério da Saúde. Relação Nacional de Medicamentos Essenciais - RENAME 2000. Brasília: Ministério da Saúde, 2000.

20. Brasil. Ministério da Saúde. Relação Nacional de Medicamentos Essenciais - RENAME 2002. Brasília: Ministério da Saúde, 2002.

21. Brasil. Ministério da Saúde. Relação Nacional de Medicamentos Essenciais - RENAME 2006. Brasília: Ministério da Saúde, 2007.

22. Brasil. Ministério da Saúde. Relação Nacional de Medicamentos Essenciais - RENAME 2008 (Série B. Textos Básicos de Saúde). Brasília: Ministério da Saúde, 2009.

23. Brasil. Ministério da Saúde. Secretaria de Ciência, Tecnologia e Insumos Estratégicos. Departamento de Assistência Farmacêutica e Insumos Estratégicos. Formulário Terapêutico Nacional 2010 (Série B. Textos Básicos de Saúde). Brasília: Ministério da Saúde, 2008. 897 p.

24. Brasil. Ministério da Saúde. Secretaria de Ciência, Tecnologia e Insumos Estratégicos. Departamento de Assistência Farmacêutica e Insumos Estratégicos. Formulário Terapêutico Nacional 2010 (Série B. Textos Básicos de Saúde). Rename 2010. Brasília: Ministério da Saúde, 2010. $1140 \mathrm{p}$

25. Wannmacher L. Quanto é evidente a evidência na saúde? Uso Racional de Medicamentos - Temas selecionados. Brasília, v. 3, n. 5, abr. 2006.

26. World Health Organization Collaborating Centre for Drugs Statistics Methodology. Anatomical Therapeutic Chemical Classification (ATC code). Disponível em: http://www.whocc.no/ atcddd. Acesso em: 06 jul. 2009.

27. Brasil. Ministério da Saúde. Portaria GM/MS no 204/2007. Regulamenta o financiamento e a transferência dos recursos federais para as ações e os serviços de saúde, na forma de blocos de financiamento, com o respectivo monitoramento e controle. Diário Oficial da União de 29 de janeiro de 2007.

28. Brasil. Ministério da Saúde. Portaria GM/MS no 2.981/2009. Aprova o Componente Especializado da Assistência Farmacêutica. Diário Oficial da União de 26 de novembro de 2009.

29. Brasil. Ministério da Saúde. Portaria GM/MS 4.217/2010. Aprova as normas de financiamento e execução do componente Básico da Assistência Farmacêutica. Diário Oficial da União de 29 de dezembro de 2010 .

30. Schedlbauer A, Prasad V, Mulvaney C, Phansalkar S, Stanton W, Bates DW, et al. What evidence supports the use of computerized alerts and prompts to improve clinicians' prescribing behavior? J Am Med Infor Assoc. 2006;16(4):531-8. 